\title{
An Overview of Systematic Reviews of Randomized-Controlled Trials for Preventing Necrotizing Enterocolitis in Preterm Infants
}

\author{
Tao Xionga, ${ }^{a}$ Akhil Maheshwari ${ }^{d}$ Josef $\mathrm{Neu}^{\mathrm{e}}$ Ahmed El-Saie $^{c}$ \\ Mohan Pammic \\ a Department of Pediatrics, West China Second University Hospital, Sichuan University, Chengdu, China; \\ ${ }^{b}$ Key Laboratory of Birth Defects and Related Diseases of Women and Children (Sichuan University), \\ Ministry of Education, Chengdu, China; ' $\mathrm{D}$ epartment of Pediatrics, Section of Neonatology, Baylor College of \\ Medicine and Texas Children's Hospital, Houston, TX, USA; division of Neonatology, Department of \\ Pediatrics, Johns Hopkins University, Baltimore, MD, USA; ${ }^{~}$ Department of Pediatrics, Section of Neonatology, \\ University of Florida, Gainesville, FL, USA
}

\section{Keywords}

Necrotizing enterocolitis · Overview ·

Randomized-controlled trial · Preterm · Neonate

\begin{abstract}
Necrotizing enterocolitis (NEC) remains a major cause for neonatal mortality and morbidity in preterm infants. The purpose of this review was to summarize evidence from systematic reviews of randomized-controlled trials (RCTs) regarding antenatal and postnatal interventions for preventing NEC. PubMed, EMBASE, the Cochrane Library Databases, Database of Abstracts of Reviews of Effects, and Campbell Library were searched for meta-analyses in which NEC was reported as an outcome after antenatal or postnatal strategies. The AMSTAR instrument was used to evaluate quality of included reviews. Grading of Recommendations, Assessment, Development and Evaluation assessment was used to evaluate certainty of evidence. We identified 98 meta-analyses of RCTs. The quality of included reviews was adequate, whereas the certainty of evidence was moderate to very low. Moderate certainty evidence showed a reduction in NEC fol-
\end{abstract}

\section{KARGER}

() 2019 S. Karger AG, Basel

karger@karger.com

www.karger.com/neo lowing administration of a combination of species of probiotics, probiotics (any), antenatal corticosteroids in pregnant women at risk of preterm birth, and ibuprofen versus indomethacin for treatment of patent ductus arteriosus (PDA). For surgical NEC, moderate certainty evidence showed an increased risk with lower (85-89\%) oxygen saturation target levels, compared with higher (91-95\%) oxygen saturation target levels. In conclusion, decreased risk of NEC is observed with probiotics, antenatal corticosteroids for women at risk of preterm birth, and ibuprofen versus indomethacin for treatment of PDA. Targeting lower oxygen saturations may increase the risk of surgical NEC, compared to targeting higher saturations.

(c) 2019 S. Karger AG, Basel

\section{Introduction}

Necrotizing enterocolitis (NEC) is a devastating gastrointestinal disease of preterm infants [1]. Despite advances in neonatal care, morbidity and mortality related to NEC have not declined for decades [2]. The incidence 
of NEC ranges between 5 and $10 \%$ in very low birthweight infants (birth weight $<1,500 \mathrm{~g}$ ), and mortality is high (about 25\%) [3]. Up to half of infants with NEC require surgical intervention, and survivors may suffer from short-gut syndrome, total parenteral nutrition dependency, cholestatic liver disease related to total parenteral nutrition administration, and neurodevelopmental delay among others [2]. The etiology of NEC is not clear but likely to be multifactorial. Demonstrated risk factors for NEC include gut immaturity, formula feeding, microbial dysbiosis, and infection [4-6]. Studies on the microbiome of the gut preceding NEC have shown an increase in phylum Proteobacteria and decrease in phyla Firmicutes and Bacteroidetes [7]. Health-care costs in the United States due to NEC are high, and the additional expenditure has been calculated to be USD 74,004 for NEC and USD 198,040 for surgical NEC per infant, respectively (based on 2011 estimations) [8].

Since the pathogenesis of NEC is multifactorial, many interventions to prevent NEC targeting various aspects of NEC etiopathogenesis have been studied. The interventions for preventing NEC include optimal feeding strategies including donor human milk [4, 9-11], microbial optimization strategies (probiotics, prebiotics, synbiotics, and antibiotics) [12-14], immunomodulatory strategies [15-17] (lactoferrin, pentoxifylline, oral immunoglobulins), and nutritional strategies [18] (glutamine and arginine supplementation) among others. The wide range of interventions reflects the need to address the multifactorial etiology of NEC.

NEC is the targeted primary outcome for NEC prevention studies but may also be an important secondary outcome for interventions focused on other disease conditions (such as surfactant for prevention of respiratory distress syndrome) [19]. The impact of antenatal interventions on NEC is often not studied or reported, for example, interventions to prevent preterm birth in women with preterm labor [20] or prolonged rupture of membranes [21]. Considering the large numbers of systematic reviews that have been published on prevention of NEC, an overview of existing systematic reviews is justified to provide an overall perspective for the bedside clinician and researchers.

\section{Methods}

\section{Search Strategy}

Two reviewers (T.X. and M.P.) searched PubMed, EMBASE, the Cochrane Database of Systematic Reviews, Database of Abstracts of Reviews of Effects, and Campbell Library from inception to August
4, 2018, for meta-analyses of randomized control trials (RCTs) for preventing NEC in preterm infants. We used the following search strategy: (necrotizing enterocolitis OR necrotizing enterocolitis OR NEC) AND (systematic review OR meta-analysis) as subject word and random word for all fields (online suppl. Table 1; for all online suppl. material, see www.karger.com/doi/10.1159/000504371). Two reviewers (T.X. and M.P.) independently screened the titles and abstracts and selected articles for full-text review and potentially eligible articles were retrieved for perusal in full text. We resolved disagreements through discussion, or, if required, we consulted a third member (A.E.-S.) of the review team.

\section{Selection Criteria}

We followed the editorial decision tree proposed by the "Cochrane Comparing Multiple Interventions methods Group" to establish whether our review would better fit an overview format or an intervention review format [22]. We did not aim to compare multiple interventions to obtain their comparative effectiveness or rank the interventions, and the overview format was the best strategy (online suppl. Fig. 1).

We included meta-analyses of RCTs where a summary estimate (risk ratio [RR], OR, or risk difference with 95\% CI) were reported. If a systematic review included both observational studies and RCTs, we included data from RCTs only. If 2 or more systematic reviews were published for the same prevention and same outcome, we selected the one with the highest number of RCT studies and the highest AMSTAR score. For updated Cochrane reviews, we selected the latest update.

We included reviews if the target population is at least one of the following: (1) preterm ( $<37$ completed weeks of gestation) neonates ( $<28$ days) or (2) low birth weight $(<2,500 \mathrm{~g})$ (including very low birthweight $[<1,500 \mathrm{~g}]$ infants and extremely low birthweight $[<1,000 \mathrm{~g}]$ infants). We considered all types of antenatal and postnatal interventions compared with placebo, no treatment, or an alternative intervention that reported NEC as a primary or a secondary outcome. We included both pharmacological and nonpharmacological interventions. Our primary outcome was incidence of NEC (stage II or above; any stage) based on modified Bell's criteria [23] during hospital stay. Secondary outcomes were (1) surgical NEC (i.e., Bell's stage III NEC, including placement of peritoneal drains), (2) "all-cause mortality" of infants during hospital stay (only from reviews that NEC was primary outcome), and (3) NEC-related mortality. We only included systematic reviews published in English. We also included interventions that increased the risk of NEC to emphasize avoidance of those interventions. We excluded network meta-analyses, where direct head to head comparison of different interventions was performed.

\section{Data Collection and Analysis}

T.X. and M.P. independently extracted data from eligible systematic reviews. From each meta-analysis, we extracted (1) the first author, year of publication, (2) number of trials and neonates and their characteristics, (3) quality of the included trials (as reported by the review authors), (4) interventions and comparisons relevant to this overview, (5) outcomes relevant to this overview (including definitions), (6) summary estimate of the intervention effects, (7) heterogeneity index including $\mathrm{Tau}^{2}, p$ value for chi-square and $I^{2}$, and (8) any other characteristics required to assess AMSTAR and Grading of Recommendations, Assessment, Development and Evaluation (GRADE) score. 


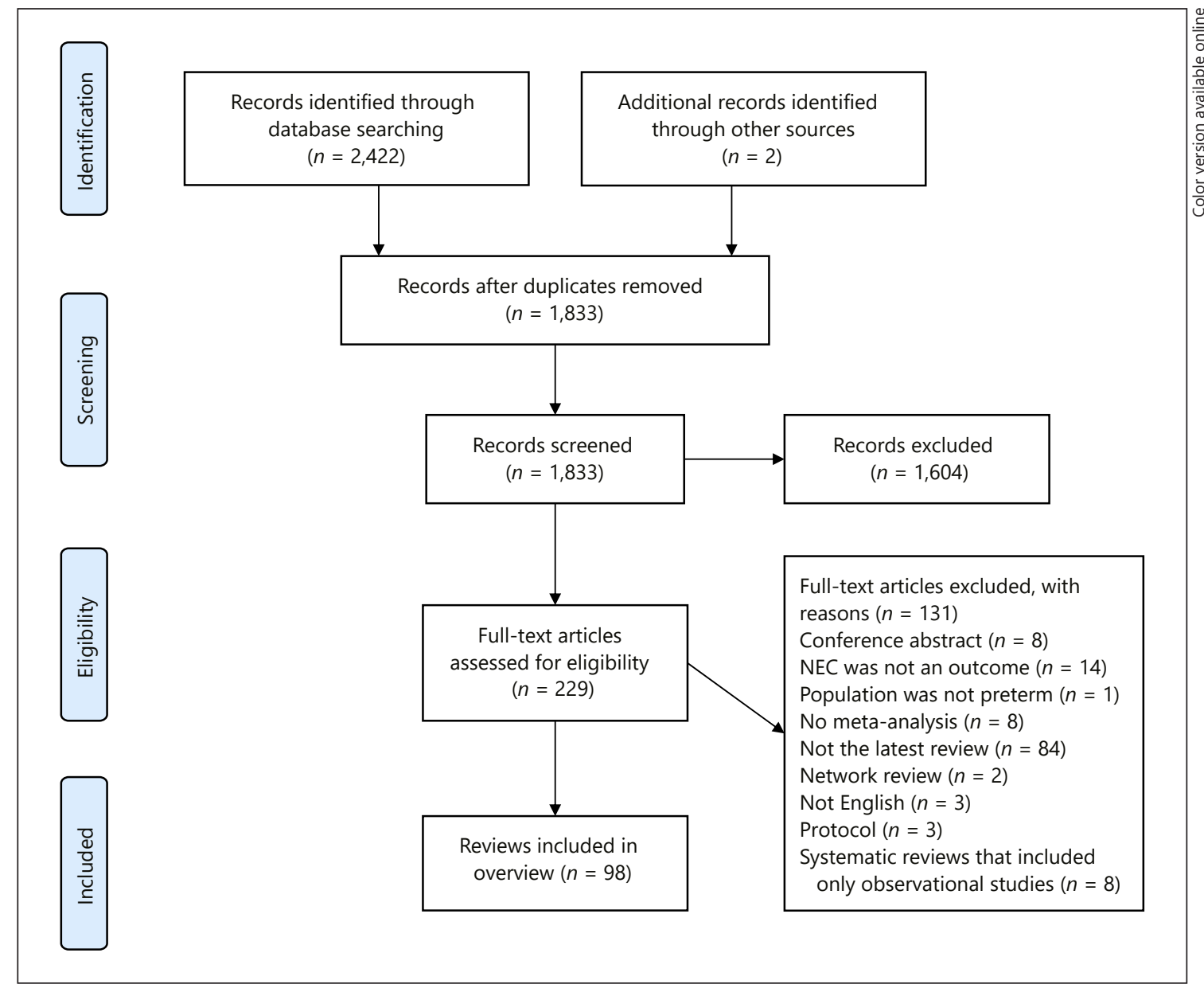

Fig. 1. Flowchart of selection of studies for inclusion in umbrella review (PRISMA 2009 flow diagram). NEC, necrotizing enterocolitis.

\section{Quality of Included Reviews}

The methodological quality of each systematic review was evaluated using a structured tool, the AMSTAR (A Measurement Tool to Assess Systematic Reviews) instrument [24]. AMSTAR evaluates the systematic review using 11 distinct criteria. Each criterion of AMSTAR has 4 choices, namely, "yes" (clearly done), "no" (clearly not done), "cannot answer," or "not applicable." We graded a review that met all of the 11 criteria to be the highest quality; achieved scores of 8-11 as high quality; scores of 4-7 as medium quality; and scores of $0-3$ as low quality [25].

\section{Certainty of Studies Included within Reviews}

Two authors (T.X. and M.P.) independently assessed the certainty of evidence and used GradePro software to enter data and assess GRADE. In the GRADE approach, outcomes from RCTs start as high-certainty evidence [26]. Relevant evidence can be rated down for high risk of bias of included studies, indirectness, imprecision, inconsistency, and publication bias. The GRADE tool assesses the certainty of evidence for each outcome by categorizing evidence into "high," "moderate," "low," or "very low" certainty [26]. There was an inherent subjectivity in assessing the certainty of evidence, and the original review authors may overstate the evidence [27]. Although some review authors have provided the GRADE assessment, we reassessed the certainty of evidence by information provided by original systematic review and derived GRADE certainty of evidence for the specified outcomes of this overview, which made a more consistent assessment for certainty of evidence.

\section{Data Synthesis}

We reported summary estimates of the interventions from included reviews qualitatively without pooling data from the included meta-analyses. For better visual presentation, we constructed forest plots using Microsoft Excel from the extracted relative effect and $95 \%$ CIs for the 4 specified outcomes.

According to the certainty of evidence (GRADE) and the summary estimates reported by the review, we graded interventions for NEC into 5 categories [25]: (1) effective interventions: high-certainty evidence of benefit, (2) possibly effective interventions (more evidence needed): moderate-certainty evidence of benefit, (3) ineffective interventions: high-certainty evidence with lack of benefit, or harm, (4) probably ineffective interventions (more evi- 
Table 1. Interventions for prevention of NEC with moderate certainty evidence

\begin{tabular}{|c|c|c|c|c|c|c|c|c|c|c|c|c|}
\hline Intervention & $\begin{array}{l}\text { Author, } \\
\text { year }\end{array}$ & Studies & Setting & $\begin{array}{l}\text { Events/ } \\
\text { intervention } \\
\text { Events/control }\end{array}$ & $\begin{array}{l}\text { Summary } \\
\text { of measure }\end{array}$ & $\begin{array}{l}\text { Forest } \\
\text { plot }\end{array}$ & $\begin{array}{l}\text { Estimate } \\
(95 \% \mathrm{CI})\end{array}$ & $\mathrm{Tau}^{2}$ & $\begin{array}{l}p \text { value } \\
\text { for chi- } \\
\text { square }\end{array}$ & $I^{2, \%}$ & $\begin{array}{l}\text { Amstar } \\
\text { score }\end{array}$ & $\begin{array}{l}\text { Certainty } \\
\text { of evidence } \\
\text { (GRADE) }\end{array}$ \\
\hline Probiotics $^{\text {a }}$ (a combination of species) & $\begin{array}{l}\text { Sawh } \\
\text { et al. [12], } \\
2016\end{array}$ & 18 & $\begin{array}{l}<37 \text { weeks or } \\
<2,500 \mathrm{~g}\end{array}$ & $\begin{array}{r}55 / 2,357 \\
134 / 2,293\end{array}$ & $\begin{array}{l}\text { RR } \\
\text { Random }\end{array}$ & $=$ & $\begin{array}{l}0.41 \\
(0.29-0.56)\end{array}$ & 0 & 0.8 & 0 & 9 & Moderate \\
\hline $\begin{array}{l}\text { Antenatal corticosteroids for accelerating fetal } \\
\text { lung maturation }{ }^{\mathrm{b}}\end{array}$ & $\begin{array}{l}\text { Roberts } \\
\text { et al. [28], } \\
2017\end{array}$ & 10 & $\begin{array}{l}\text { Women at risk } \\
\text { of preterm birth }\end{array}$ & $\begin{array}{l}27 / 2,380 \\
53 / 2,322\end{array}$ & $\begin{array}{l}\text { RR } \\
\text { Fixed }\end{array}$ & 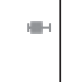 & $\begin{array}{l}0.50 \\
(0.32-0.78)\end{array}$ & $\begin{array}{l}\text { No } \\
\text { info }\end{array}$ & 0.7 & 0 & 11 & Moderate \\
\hline $\begin{array}{l}\text { Probiotics } \\
\text { (Any) }\end{array}$ & $\begin{array}{l}\text { Sawh } \\
\text { et al. [12], } \\
2016\end{array}$ & 38 & $\begin{array}{l}<37 \text { weeks or } \\
<2,500 \mathrm{~g}\end{array}$ & $\begin{array}{l}170 / 5,304 \\
311 / 5,216\end{array}$ & $\begin{array}{l}\text { RR } \\
\text { Random }\end{array}$ & - & $\begin{array}{l}0.53 \\
(0.42-0.66)\end{array}$ & 0.04 & 0.29 & 11 & 9 & Moderate \\
\hline $\begin{array}{l}\text { Ibuprofen for the treatment of } \mathrm{PDA}^{\mathrm{b}, \mathrm{c}} \\
\text { (Ibuprofen vs. indomethacin) }\end{array}$ & $\begin{array}{l}\text { Ohlsson } \\
\text { et al. [29], } \\
2015\end{array}$ & 16 & $\begin{array}{l}<37 \text { weeks and/or } \\
<2,500 \mathrm{~g} \text { with } \\
\text { PDA }\end{array}$ & $\begin{array}{l}38 / 489 \\
58 / 459\end{array}$ & $\begin{array}{l}\text { RR } \\
\text { Fixed }\end{array}$ & 齓- & $\begin{array}{l}0.64 \\
(0.45-0.93)\end{array}$ & $\begin{array}{l}\text { No } \\
\text { info }\end{array}$ & 0.87 & 0 & 10 & Moderate \\
\hline $\begin{array}{l}\text { Early }(<8 \text { days) postnatal corticosteroids for } \\
\text { preventing chronic lung disease }{ }^{b}\end{array}$ & $\begin{array}{l}\text { Doyle } \\
\text { et al. [30], } \\
2014\end{array}$ & 23 & $\begin{array}{l}\text { Preterm infants } \\
\text { at risk of } \\
\text { developing } \\
\text { chronic } \\
\text { lung disease }\end{array}$ & $\begin{array}{l}142 / 1,764 \\
161 / 1,743\end{array}$ & $\begin{array}{l}\mathrm{RR} \\
\text { Fixed }\end{array}$ & 是 & $\begin{array}{l}0.87 \\
(0.70-1.08)\end{array}$ & $\begin{array}{l}\text { No } \\
\text { info }\end{array}$ & 0.9 & 0 & 10 & Moderate \\
\hline $\begin{array}{l}\text { Early erythropoietin for preventing red blood } \\
\text { cell transfusion }{ }^{\mathrm{b}}\end{array}$ & $\begin{array}{l}\text { Ohlsson and } \\
\text { Aher [31], } \\
2014\end{array}$ & 11 & $\begin{array}{l}<37 \text { weeks and/or } \\
\text { low }<2,500 \mathrm{~g}, \\
<8 \text { days of age }\end{array}$ & $\begin{array}{l}52 / 678 \\
45 / 669\end{array}$ & $\begin{array}{l}\text { RR } \\
\text { Fixed }\end{array}$ & & $\begin{array}{l}1.07 \\
(0.73-1.57)\end{array}$ & $\begin{array}{l}\text { No } \\
\text { info }\end{array}$ & 0.77 & 0 & 10 & Moderate \\
\hline \multirow[t]{2}{*}{$\begin{array}{l}\text { Prophylactic intravenous indomethacin }{ }^{\mathrm{b}} \\
\text { (prophylactic indomethacin vs. control) }\end{array}$} & $\begin{array}{l}\text { Fowlie } \\
\text { Peter et al. } \\
\text { [32], } 2010\end{array}$ & 12 & $<37$ weeks & $\begin{array}{l}84 / 1,187 \\
77 / 1,214\end{array}$ & $\begin{array}{l}\text { RR } \\
\text { Fixed }\end{array}$ & & $\begin{array}{l}1.09 \\
(0.82-1.46)\end{array}$ & $\begin{array}{l}\text { No } \\
\text { info }\end{array}$ & 0.88 & 0 & 9 & Moderate \\
\hline & & & & & & & & & & & & \\
\hline
\end{tabular}

NEC, necrotizing enterocolitis; PDA, patent ductus arteriosus; RR, risk ratio; GRADE, Grading of Recommendations, Assessment, Development and Evaluation. ${ }^{a}$ Primary outcome; ${ }^{b}$ secondary outcome; ${ }^{c}$ NEC at any stage (stages $1,2,3)$

dence needed): moderate-certainty evidence with lack of benefit, or harm, and (5) no conclusions possible: low or very low certainty evidence, or insufficient evidence to comment on the effectiveness of an intervention.

\section{Results}

The process of including reviews for our overview is depicted in Figure 1. The search yielded 2,422 articles from databases and 2 from other sources. Discarding the duplicates and excluding unrelated articles by title and abstract, we had 229 reviews for which full texts of the articles were obtained. Finally, we included 98 reviews after excluding 131 reviews. NEC was targeted as a primary outcome in 22 reviews and as a secondary outcome in 76 reviews. NEC was reported as an outcome in 110 interventions, surgical NEC in 10 interventions, "all-cause mortality" during hospital stay in 21 interventions, and NEC-related mortality in 4 interventions.

\section{Effect of Interventions}

Outcome 1: NEC (Stage II or Above; Any Stage)

(1) Effective interventions: None.

(2) Possibly effective interventions: (Table 1):

Preventing NEC in Preterm Infants: An

Overview
Moderate certainty evidence showed a reduction in NEC following administration of a combination of species of probiotics (RR $0.41,95 \%$ CI $0.29-0.56$; 18 trials; 4,650 infants) [12]; antenatal corticosteroids for accelerating fetal lung maturation in pregnant women at risk of preterm birth (RR 0.50, 95\% CI 0.32-0.78; 10 trials; 4,702 infants) [28]; probiotics (any; RR $0.53,95 \%$ CI $0.42-0.66 ; 38$ trials; 10,520 infants) [12]; and ibuprofen compared to indomethacin for the treatment of patent ductus arteriosus (PDA; RR 0.64, 95\% CI 0.45-0.93; 16 trials; 948 infants) [29].

(3) Ineffective interventions: None.

(4) Probably ineffective interventions: (Table 1):

A. Harm effect: None.

B. Lack of effectiveness:

Moderate certainty evidence showed lack of effectiveness in NEC by early ( $<8$ days) postnatal corticosteroids for preventing chronic lung disease (RR $0.87,95 \% \mathrm{CI}$ $0.70-1.08$; 23 trials; 3,507 infants) [30]; early erythropoietin for preventing red blood cell transfusion (RR 1.07, 95\% CI 0.73-1.57; 11 trials; 1,347 infants) [31]; and prophylactic intravenous indomethacin (RR 1.09, 95\% CI 0.82-1.46; 12 trials; 2,401 infants) [32].

(5) No conclusions possible because of low (online suppl. Fig. 2) or very low certainty evidence (online suppl. Fig. 3) in 103 preventative interventions for NEC. 
Table 2. Interventions for prevention of surgical NEC

\begin{tabular}{|c|c|c|c|c|c|c|c|c|c|c|c|c|c|}
\hline Intervention & $\begin{array}{l}\text { Author } \\
\text { year }\end{array}$ & Studies & Setting & $\begin{array}{l}\text { Events/ } \\
\text { intervention } \\
\text { events/control }\end{array}$ & $\begin{array}{l}\text { Summary } \\
\text { of measure }\end{array}$ & $\begin{array}{l}\text { Forest } \\
\text { plot }\end{array}$ & & $\begin{array}{l}\text { Estimate } \\
(95 \% \mathrm{CI})\end{array}$ & $\mathrm{Tau}^{2}$ & $\begin{array}{l}p \text { value } \\
\text { for chi- } \\
\text { square }\end{array}$ & $I^{2}, \%$ & $\begin{array}{l}\text { Amstar } \\
\text { score }\end{array}$ & $\begin{array}{l}\text { Certainty } \\
\text { of evidence } \\
\text { (GRADE) }\end{array}$ \\
\hline $\begin{array}{l}\text { Oxygen Saturation Target Levels }{ }^{\mathrm{b}} \\
\text { (lower [85-89\%] vs. higher [91-95\%] } \mathrm{SPO}_{2} \text { ) }\end{array}$ & $\begin{array}{l}\text { Askie } \\
\text { et al. [33], } \\
2018\end{array}$ & 5 & $\begin{array}{l}<28 \text { weeks } \\
\text { within } 24 \mathrm{~h} \\
\text { of birth }\end{array}$ & $\begin{array}{l}227 / 2,464 \\
170 / 2,465\end{array}$ & $\begin{array}{l}\text { RR } \\
\text { Fixed }\end{array}$ & & 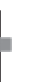 & $\begin{array}{l}1.33 \\
(1.10-1.61)\end{array}$ & No info & no info & 0 & 5 & Moderate \\
\hline Arginine supplementation ${ }^{\mathrm{a}}$ & $\begin{array}{l}\text { Shah } \\
\text { et al. [36], } \\
2017\end{array}$ & 3 & $<37$ weeks & $\begin{array}{l}1 / 140 \\
8 / 145\end{array}$ & $\begin{array}{l}\text { RR } \\
\text { Fixed }\end{array}$ & $\mapsto$ & & $\begin{array}{l}0.13 \\
(0.02-1.03)\end{array}$ & No info & no info & 0 & 10 & Low \\
\hline Donor-banked milk vs. formula ${ }^{a}$ & $\begin{array}{l}\text { Silano } \\
\text { et al. [37], } \\
2018\end{array}$ & 4 & $\begin{array}{l}<37 \text { weeks } \\
\text { and/or } \\
<2,500 \mathrm{~g}\end{array}$ & $\begin{array}{l}10 / 469 \\
25 / 484\end{array}$ & $\begin{array}{l}\text { OR } \\
\text { Random }\end{array}$ & $r$ & & $\begin{array}{l}0.45 \\
(0.19-1.09)\end{array}$ & No info & 0.29 & 20.68 & 8 & Low \\
\hline Probiotics ${ }^{\mathrm{a}}$ (Any) & $\begin{array}{l}\text { Rees } \\
\text { et al. [38], } \\
2017\end{array}$ & 14 & $\begin{array}{l}\text { Premature } \\
\text { infants }\end{array}$ & $\begin{array}{l}48 / 1,989 \\
74 / 1,986\end{array}$ & $\begin{array}{l}\text { RR } \\
\text { Random }\end{array}$ & 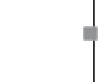 & & $\begin{array}{l}0.74 \\
(0.51-1.05)\end{array}$ & No info & 0.73 & 0 & 9 & Low \\
\hline $\begin{array}{l}\text { Antistaphylococcal immunoglobulins to } \\
\text { prevent staphylococcal infection }^{\mathrm{b}}\end{array}$ & $\begin{array}{l}\text { Shah } \\
\text { et al. [39], } \\
2009\end{array}$ & 2 & $\begin{array}{l}\text { Very low birth } \\
\text { weight infants }\end{array}$ & $\begin{array}{l}27 / 1,341 \\
24 / 1,147\end{array}$ & $\begin{array}{l}\mathrm{RR} \\
\text { Fixed }\end{array}$ & 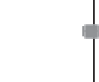 & & $\begin{array}{l}0.80 \\
(0.46-1.40)\end{array}$ & No info & 0.57 & 0 & 9 & Low \\
\hline $\begin{array}{l}\mathrm{N} \text {-acetylcysteine supplementation in } \\
\text { parenterally fed neonates }{ }^{\mathrm{b}}\end{array}$ & $\begin{array}{l}\text { Soghier } \\
\text { et al. [40], } \\
2006\end{array}$ & 1 & $\begin{array}{l}\text { Neonates } \\
\leq \text { minimal } \\
\text { enteral nutrition }\end{array}$ & $\begin{array}{l}17 / 194 \\
16 / 197\end{array}$ & RR & & 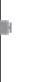 & $\begin{array}{l}1.08 \\
(0.56-2.07)\end{array}$ & $\mathrm{n} / \mathrm{a}$ & $\mathrm{n} / \mathrm{a}$ & $\mathrm{n} / \mathrm{a}$ & 9 & Low \\
\hline $\begin{array}{l}\text { Human milk feeding }{ }^{\mathrm{a}} \text { (exclusive human milk vs. } \\
\text { exclusive preterm formula) }\end{array}$ & $\begin{array}{l}\text { Miller } \\
\text { et al. [41], } \\
2018\end{array}$ & 1 & $\begin{array}{l}\leq 28 \text { weeks } \\
\text { and/or } \\
\leq 1,500 \mathrm{~g}\end{array}$ & $\begin{array}{l}0 / 29 \\
4 / 24\end{array}$ & $\begin{array}{l}\text { RR } \\
\text { Random }\end{array}$ & $\longmapsto$ & & $\begin{array}{l}0.09 \\
(0.01-1.64)\end{array}$ & $\mathrm{n} / \mathrm{a}$ & $\mathrm{n} / \mathrm{a}$ & $\mathrm{n} / \mathrm{a}$ & 8 & Very low \\
\hline $\begin{array}{l}\text { Human milk feeding (unpasteurised vs. } \\
\text { pasteurised human milk) }\end{array}$ & $\begin{array}{l}\text { Miller } \\
\text { et al. [41], } \\
2018\end{array}$ & 1 & $\begin{array}{l}\leq 28 \text { weeks } \\
\text { and/or } \\
\leq 1,500 \mathrm{~g}\end{array}$ & $\begin{array}{l}0 / 151 \\
4 / 152\end{array}$ & $\begin{array}{l}\text { RR } \\
\text { Random }\end{array}$ & $\longmapsto$ & H & $\begin{array}{l}0.11 \\
(0.01-2.06)\end{array}$ & $\mathrm{n} / \mathrm{a}$ & $\mathrm{n} / \mathrm{a}$ & $\mathrm{n} / \mathrm{a}$ & 8 & Very low \\
\hline Oral immunoglobulin ${ }^{\mathrm{a}}$ & $\begin{array}{l}\text { Foster } \\
\text { et al. [17], } \\
2016\end{array}$ & 2 & $\begin{array}{l}<37 \text { weeks } \\
\text { or }<2,500 \text { g }\end{array}$ & $\begin{array}{l}0 / 153 \\
4 / 158\end{array}$ & $\mathrm{RR}$ & $\longmapsto$ & & $\begin{array}{l}0.21 \\
(0.02-1.75)\end{array}$ & No info & 0.7 & 0 & 9 & Very low \\
\hline $\begin{array}{l}\text { Human milk feeding }{ }^{\mathrm{a}} \text { (higher vs. lower dose } \\
\text { human milk) }\end{array}$ & $\begin{array}{l}\text { Miller } \\
\text { et al. [41], } \\
2018\end{array}$ & 2 & $\begin{array}{l}\leq 28 \text { weeks } \\
\text { and/or } \\
\leq 1,500 \mathrm{~g}\end{array}$ & $\begin{array}{r}6 / 321 \\
12 / 259\end{array}$ & $\begin{array}{l}\text { RR } \\
\text { Random }\end{array}$ & 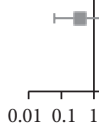 & 10 & $\begin{array}{l}0.36 \\
(0.06-2.04)\end{array}$ & 1.02 & 0.09 & 66 & 8 & Very low \\
\hline
\end{tabular}

NEC, necrotizing enterocolitis; RR, risk ratio; GRADE, Grading of Recommendations, Assessment, Development and Evaluation. ${ }^{\text {a }}$ Primary outcome; ${ }^{\text {b }}$ secondary outcome.

Outcome 2: Surgical NEC (Including Placement of Peritoneal Drains; Table 2)

(1) Effective interventions: None.

(2) Possibly effective interventions: None.

(3) Ineffective interventions: None.

(4) Probably ineffective interventions:

A. Harm effect:

Moderate certainty evidence showed an increase in surgical NEC by lower (85-89\%) vs. higher (91-95\%) oxygen saturation target levels (RR 1.33, 95\% CI 1.10-1.61; 5 trials; 4,929 infants) [33].

B. Lack of effectiveness: None.

(5) No conclusions possible:

No conclusion could be drawn because of low to very low certainty evidence for arginine supplementation, donor-banked milk versus formula, probiotics (any), antistaphylococcal immunoglobulins to prevent staphylococcal infection, $\mathrm{N}$-acetylcysteine supplementation in parenterally fed neonates, exclusive human milk versus exclusive preterm formula, unpasteurized versus pasteur- ized human milk, oral immunoglobulin, and higher versus lower dose human milk.

Outcome 3: "All-Cause Mortality" during Hospital Stay (Table 3)

(1) Effective interventions: None.

(2) Possibly effective interventions:

Moderate certainty evidence review showed a reduction in mortality following a combination of species of probiotics (RR $0.41,95 \%$ CI $0.29-0.56 ; 18$ trials; 4,650 infants) and probiotics (any; RR 0.79, 95\% CI 0.68-0.93; 29 trials; 9,507 infants) [12].

(3) Ineffective interventions: None.

(4) Probably ineffective interventions:

A. Harm effect: None.

B. Lack of effectiveness: None.

(5) No conclusions possible

No conclusion could be drawn for probiotics (single species Lactobacillus), arginine supplementation; antibiotics for preterm rupture of membranes (any antibiotic 
Table 3. Effect of interventions for prevention of NEC as primary outcome on "all-cause mortality" during hospital stay

\begin{tabular}{|c|c|c|c|c|c|c|c|c|c|c|c|c|}
\hline Intervention & $\begin{array}{l}\text { Author } \\
\text { year }\end{array}$ & Studies & Setting & $\begin{array}{l}\text { Events/ } \\
\text { intervention } \\
\text { Events/control }\end{array}$ & $\begin{array}{l}\text { Summary } \\
\text { of measure }\end{array}$ & $\begin{array}{l}\text { Forest } \\
\text { plot }\end{array}$ & $\begin{array}{l}\text { Estimate } \\
(95 \% \mathrm{CI})\end{array}$ & $\mathrm{Tau}^{2}$ & $\begin{array}{l}p \text { value } \\
\text { for chi- } \\
\text { square }\end{array}$ & $I^{2, \%}$ & $\begin{array}{l}\text { Amstar } \\
\text { score }\end{array}$ & $\begin{array}{l}\text { Certainty } \\
\text { of evidence } \\
\text { (GRADE) }\end{array}$ \\
\hline Probiotics ${ }^{\mathrm{a}}$ (a combination of species) & $\begin{array}{l}\text { Sawh et al. } \\
{[12], 2016}\end{array}$ & 18 & $\begin{array}{l}<37 \text { weeks or } \\
<2,500 \mathrm{~g}\end{array}$ & $\begin{array}{r}55 / 2,357 \\
134 / 2,293\end{array}$ & $\begin{array}{l}\text { RR } \\
\text { Random }\end{array}$ & $=$ & $\begin{array}{l}0.41 \\
(0.29-0.56)\end{array}$ & 0 & 0.8 & 0 & 9 & Moderate \\
\hline Probiotics $^{\mathrm{a}}$ (any) & $\begin{array}{l}\text { Sawh et al. } \\
{[12], 2016}\end{array}$ & 29 & $\begin{array}{l}<37 \text { weeks or } \\
<2,500 \mathrm{~g}\end{array}$ & $\begin{array}{l}264 / 4,761 \\
341 / 4,746\end{array}$ & $\begin{array}{l}\text { RR } \\
\text { Random }\end{array}$ & + & $\begin{array}{l}0.79 \\
(0.68-0.93)\end{array}$ & 0 & 0.59 & 0 & 9 & Moderate \\
\hline Probiotics $^{\mathrm{a}}$ (lactobacillus) & $\begin{array}{l}\text { Sawh et al. } \\
\text { [12], } 2016\end{array}$ & 8 & $\begin{array}{l}<37 \text { weeks or } \\
<2,500 \mathrm{~g}\end{array}$ & $\begin{array}{l}32 / 1,299 \\
53 / 1,297\end{array}$ & $\begin{array}{l}\text { RR } \\
\text { Random }\end{array}$ & 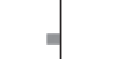 & $\begin{array}{l}0.61 \\
(0.40-0.95)\end{array}$ & 0 & 0.84 & 0 & 9 & Low \\
\hline Arginine supplementation $^{\mathrm{a}}$ & $\begin{array}{l}\text { Shah et al. } \\
{[36], 2017}\end{array}$ & 3 & $<37$ weeks & $\begin{array}{l}13 / 140 \\
18 / 145\end{array}$ & $\begin{array}{l}\text { RR } \\
\text { Fixed }\end{array}$ & th & $\begin{array}{l}0.77 \\
(0.41-1.45)\end{array}$ & $\begin{array}{l}\text { No } \\
\text { info }\end{array}$ & 0.18 & 42 & 9 & Low \\
\hline $\begin{array}{l}\text { Antibiotics for preterm rupture of membranes } \\
\text { (any antibiotic vs. placebo) }\end{array}$ & $\begin{array}{l}\text { Kenyon et al. } \\
{[42], 2013}\end{array}$ & 12 & $\begin{array}{l}\text { Women with } \\
\text { preterm rupture } \\
\text { of membranes }\end{array}$ & $\begin{array}{l}276 / 4,315 \\
138 / 1,986\end{array}$ & $\begin{array}{l}\text { RR } \\
\text { Random }\end{array}$ & 1 & $\begin{array}{l}0.93 \\
(0.76-1.14)\end{array}$ & 0 & 0.65 & 0 & 10 & Low \\
\hline Probiotics ${ }^{\mathrm{a}}$ (bifidobacterium) & $\begin{array}{l}\text { Sawh et al. } \\
{[12], 2016}\end{array}$ & 6 & $\begin{array}{l}<37 \text { weeks or } \\
<2,500 \mathrm{~g}\end{array}$ & $\begin{array}{r}69 / 1,027 \\
103 / 1,029\end{array}$ & $\begin{array}{l}\mathrm{RR} \\
\text { Random }\end{array}$ & $\mathrm{H}=-$ & $\begin{array}{l}0.37 \\
(0.14-0.97)\end{array}$ & 0.73 & 0.01 & 69 & 9 & Very low \\
\hline Lactoferrin plus probiotics vs. placebo ${ }^{a}$ & $\begin{array}{l}\text { Pammi et al. } \\
{[7], 2017}\end{array}$ & 1 & $<37$ weeks & $\begin{array}{r}9 / 238 \\
18 / 258\end{array}$ & $\begin{array}{l}\text { RR } \\
\text { Fixed }\end{array}$ & int & $\begin{array}{l}0.54 \\
(0.25-1.18)\end{array}$ & $\mathrm{n} / \mathrm{a}$ & $\mathrm{n} / \mathrm{a}$ & $\mathrm{n} / \mathrm{a}$ & 9 & Very low \\
\hline Early trophic feeding vs. enteral fasting ${ }^{a}$ & $\begin{array}{l}\text { Morgan et al. } \\
{[11], 2013}\end{array}$ & 8 & $\begin{array}{l}<1,500 \text { g or } \\
<32 \text { weeks }\end{array}$ & $\begin{array}{l}23 / 278 \\
37 / 280\end{array}$ & $\begin{array}{l}\text { RR } \\
\text { Fixed }\end{array}$ & 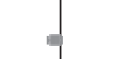 & $\begin{array}{l}0.66 \\
(0.41-1.07)\end{array}$ & $\begin{array}{l}\text { No } \\
\text { info }\end{array}$ & 0.76 & 0 & 9 & Very low \\
\hline Enteral antibiotics $^{\mathrm{a}}$ & $\begin{array}{l}\text { Bury et al. } \\
{[14], 2001}\end{array}$ & 2 & $\begin{array}{l}<2,500 \mathrm{~g} \\
\text { and } / \text { or } \\
<37 \text { weeks }\end{array}$ & $\begin{array}{l}12 / 106 \\
18 / 109\end{array}$ & $\begin{array}{l}\text { RR } \\
\text { Fixed }\end{array}$ & 虽 & $\begin{array}{l}0.67 \\
(0.34-1.32)\end{array}$ & $\begin{array}{l}\text { No } \\
\text { info }\end{array}$ & 0.25 & 24 & 9 & Very low \\
\hline $\begin{array}{l}\text { Antibiotics for preterm rupture of membranes } \\
\text { (antibiotics vs. no antibiotic [no placebo]) }\end{array}$ & $\begin{array}{l}\text { Kenyon et al. } \\
{[42], 2013}\end{array}$ & 6 & $\begin{array}{l}\text { Women with } \\
\text { preterm rupture } \\
\text { of membranes }\end{array}$ & $\begin{array}{l}23 / 289 \\
34 / 282\end{array}$ & $\begin{array}{l}\text { RR } \\
\text { Random }\end{array}$ & 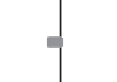 & $\begin{array}{l}0.69 \\
(0.41-1.14)\end{array}$ & 0 & 0.7 & 0 & 10 & Very low \\
\hline Probiotics ${ }^{\mathrm{a}}$ (Saccharomyces boulardii) & $\begin{array}{l}\text { Sawh et al. } \\
{[12], 2016}\end{array}$ & 2 & $\begin{array}{l}<37 \text { weeks or } \\
<2,500 \mathrm{~g}\end{array}$ & $\begin{array}{l}11 / 186 \\
13 / 171\end{array}$ & $\begin{array}{l}\text { RR } \\
\text { Random }\end{array}$ & $r$ & $\begin{array}{l}0.72 \\
(0.33-1.54)\end{array}$ & 0 & 0.63 & 0 & 9 & Very low \\
\hline $\begin{array}{l}\text { Omega-3 long-chain polyunsaturated fatty } \\
\text { acids }^{\mathrm{a}}\end{array}$ & $\begin{array}{l}\text { Zhang et al. } \\
{[43], 2014}\end{array}$ & 6 & $<37$ weeks & $\begin{array}{l}17 / 1,838 \\
26 / 1,911\end{array}$ & $\begin{array}{l}\text { RR } \\
\text { Fixed }\end{array}$ & +1 & $\begin{array}{l}0.76 \\
(0.42-1.38)\end{array}$ & $\begin{array}{l}\text { No } \\
\text { info }\end{array}$ & 0.11 & 44 & 10 & Very low \\
\hline Colostrum $^{\mathrm{a}}$ & $\begin{array}{l}\text { Sadeghirad et } \\
\text { al. [2], } 2018\end{array}$ & 7 & $\begin{array}{l}<37 \text { weeks within } \\
\text { the first week of } \\
\text { life }\end{array}$ & $\begin{array}{l}11 / 168 \\
12 / 162\end{array}$ & $\begin{array}{l}\text { RR } \\
\text { Random }\end{array}$ & $r=1$ & $\begin{array}{l}0.84 \\
(0.39-1.82)\end{array}$ & $\begin{array}{l}\text { No } \\
\text { info }\end{array}$ & 0.604 & 0 & 10 & Very low \\
\hline Prophylactic surgical ligation of $\mathrm{PDA}^{\mathrm{a}}$ & $\begin{array}{l}\text { Mosalli et al. } \\
{[44], 2009}\end{array}$ & 1 & $<1,000 \mathrm{~g}$ & $\begin{array}{l}16 / 40 \\
20 / 44\end{array}$ & $\mathrm{RR}$ & tit & $\begin{array}{l}0.88 \\
(0.53-1.45)\end{array}$ & $\mathrm{n} / \mathrm{a}$ & $\mathrm{n} / \mathrm{a}$ & $\mathrm{n} / \mathrm{a}$ & 8 & Very low \\
\hline Lactoferrin vs. placebo ${ }^{a}$ & $\begin{array}{l}\text { Pammi et al. } \\
{[7], 2017^{\mathrm{b}}}\end{array}$ & 10 & $<37$ weeks & $\begin{array}{l}127 / 2,535 \\
136 / 2,561\end{array}$ & $\begin{array}{l}\text { RR } \\
\text { Fixed }\end{array}$ & 1 & $\begin{array}{l}0.94 \\
(0.75-1.19)\end{array}$ & $\begin{array}{l}\text { No } \\
\text { info }\end{array}$ & 0.16 & 32 & 9 & Very low \\
\hline $\begin{array}{l}\text { Slow (up to } 24 \mathrm{~mL} / \mathrm{kg} / \text { days) vs. faster rates of } \\
\text { advancement of enteral feed volumes }\end{array}$ & $\begin{array}{l}\text { Oddie et al. } \\
{[4], 2017}\end{array}$ & 9 & $\begin{array}{l}\text { Very preterm } \\
\text { or }<1,500 \mathrm{~g}\end{array}$ & $\begin{array}{l}147 / 1,796 \\
127 / 1,780\end{array}$ & $\begin{array}{l}\text { RR } \\
\text { Fixed }\end{array}$ & 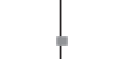 & $\begin{array}{l}1.15 \\
(0.93-1.42)\end{array}$ & $\begin{array}{l}\text { No } \\
\text { info }\end{array}$ & 0.33 & 13 & 10 & Very low \\
\hline $\begin{array}{l}\text { Delayed ( } \geq 4 \text { days) vs. early introduction of } \\
\text { progressive enteral feeding }(24 \mathrm{~mL} / \mathrm{kg} / \text { day })^{\mathrm{a}}\end{array}$ & $\begin{array}{l}\text { Morgan et al. } \\
{[11], 2013}\end{array}$ & 7 & $\begin{array}{l}<1,500 \mathrm{~g} \text { or } \\
<32 \text { weeks }\end{array}$ & $\begin{array}{l}33 / 464 \\
29 / 503\end{array}$ & $\begin{array}{l}\text { RR } \\
\text { Fixed }\end{array}$ & w & $\begin{array}{l}1.18 \\
(0.75-1.88)\end{array}$ & $\begin{array}{l}\text { No } \\
\text { info }\end{array}$ & 0.71 & 0 & 9 & Very low \\
\hline $\begin{array}{l}\text { Red blood cell transfusions }{ }^{\mathrm{a}} \text { (liberal [greater volume } \\
\text { and/or number of RBC transfusions] vs. restrictive } \\
\text { transfusion) }\end{array}$ & [45], 2016 & 8 & $<37$ weeks & $\begin{array}{l}65 / 475 \\
53 / 476\end{array}$ & $\begin{array}{l}\text { RR } \\
\text { Fixed }\end{array}$ & p & $\begin{array}{l}1.24 \\
(0.89-1.72)\end{array}$ & $\begin{array}{l}\text { No } \\
\text { info }\end{array}$ & 0.95 & 0 & 7 & Very low \\
\hline Glycerin enemas and suppositories $^{\mathrm{a}}$ & $\begin{array}{l}\text { Livingston et } \\
\text { al. [46], } 2015\end{array}$ & 3 & $\begin{array}{l}<32 \text { weeks and/or } \\
<1,500 \mathrm{~g}\end{array}$ & $\begin{array}{r}12 / 93 \\
8 / 86\end{array}$ & $\begin{array}{l}\text { RR } \\
\text { Random }\end{array}$ & $1-1$ & $\begin{array}{l}1.34 \\
(0.58-3.11)\end{array}$ & 0 & 0.82 & 0 & 9 & Very low \\
\hline Dobutamine vs. dopamine $\mathrm{e}^{\mathrm{a}}$ & $\begin{array}{l}\text { Osborn et al. } \\
{[47], 2007}\end{array}$ & 1 & $\begin{array}{l}<37 \text { weeks } \\
\text { with low } \\
\text { blood flow }\end{array}$ & $\begin{array}{r}14 / 22 \\
9 / 20\end{array}$ & $\begin{array}{l}\text { RR } \\
\text { Fixed }\end{array}$ & 监 & $\begin{array}{l}1.41 \\
(0.79-2.52)\end{array}$ & $\mathrm{n} / \mathrm{a}$ & $\mathrm{n} / \mathrm{a}$ & $\mathrm{n} / \mathrm{a}$ & 9 & Very low \\
\hline Adjunctive antibiotic treatment in preterm labor ${ }^{\mathrm{a}}$ & $\begin{array}{l}\text { Egarter et al. } \\
{[48], 1996}\end{array}$ & 4 & $\begin{array}{l}\text { Preterm labor } \\
\text { before } \\
37 \text { weeks }\end{array}$ & $\begin{array}{l}8 / 272 \\
2 / 277\end{array}$ & $\begin{array}{l}\text { OR } \\
\text { Fixed }\end{array}$ & $\longrightarrow$ & $\begin{array}{l}3.25( \\
0.93-11.38)\end{array}$ & $\begin{array}{l}\text { No } \\
\text { info }\end{array}$ & $\begin{array}{l}\text { No } \\
\text { info }\end{array}$ & $\begin{array}{l}\text { No } \\
\text { info }\end{array}$ & 4 & Very low \\
\hline
\end{tabular}

NEC, necrotizing enterocolitis; RR, risk ratio; GRADE, Grading of Recommendations, Assessment, Development and Evaluation; PDA, patent ductus arteriosus. ${ }^{a}$ Primary outcome. ${ }^{b}$ Updated with unpublished 
Table 4. Effect of interventions for prevention of NEC on NEC-related mortality

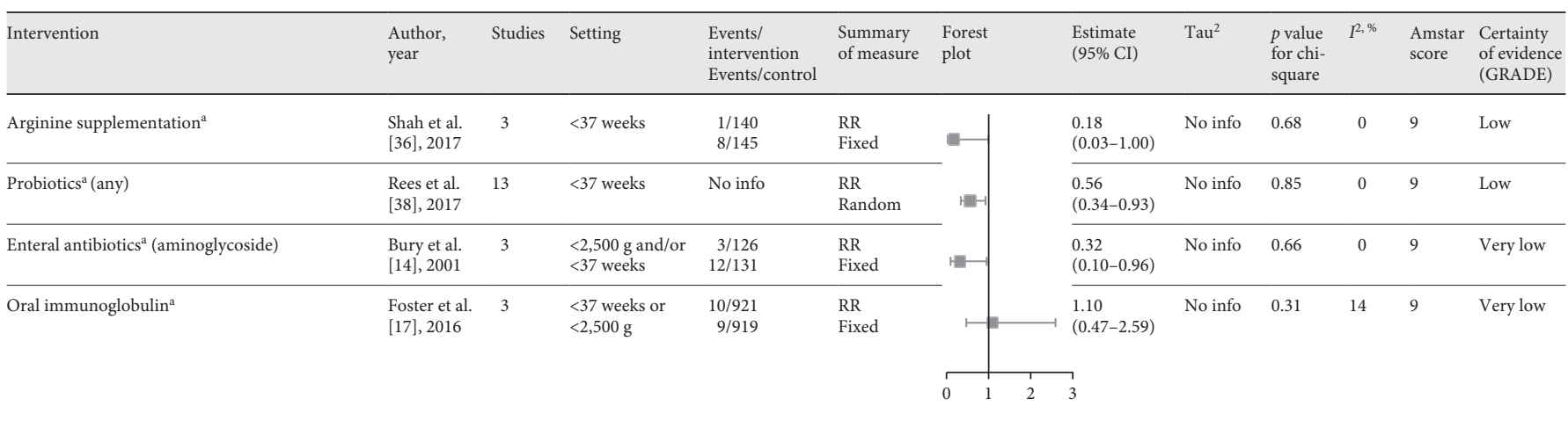

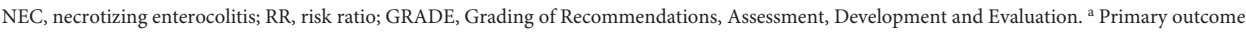

vs. placebo), probiotics (single species Bifidobacterium), lactoferrin plus probiotics versus placebo; early trophic feeding versus enteral fasting, enteral antibiotics, antibiotics for preterm rupture of membranes (antibiotics vs. no antibiotics), probiotics (single species Saccharomyces boulardii), omega-3 long-chain polyunsaturated fatty acids, colostrum, prophylactic surgical ligation of PDA, lactoferrin versus placebo, slow (up to $24 \mathrm{~mL} / \mathrm{kg}$ /day) versus faster rates of advancement of enteral feeds, delayed $(\geq 4$ days) versus early introduction of progressive enteral feeding ( $24 \mathrm{~mL} / \mathrm{kg} /$ day), red blood cell transfusions (liberal [greater volume and/or number of RBC transfusions] versus restrictive transfusion), glycerin enemas and suppositories, dobutamine versus dopamine, adjunctive antibiotic treatment in preterm labor.

Outcome 4: NEC-Related Mortality

(1) Effective interventions: None.

(2) Possibly effective interventions: None.

(3) Ineffective interventions: None.

(4) Probably ineffective interventions: None.

(5) No conclusions possible (Table 4) for arginine supplementation, probiotics (any), enteral antibiotics (aminoglycoside), and oral immunoglobulin.

\section{Heterogeneity of Included Studies}

We extracted heterogeneity index provided by the included reviews. About 4.5\% (5 of 110) of interventions had significant heterogeneity $\left(I^{2}>50 \%\right)$ for NEC, $10 \%$ ( 1 of 10) interventions had significant heterogeneity for surgical NEC, $4.7 \%$ ( 1 of 21 ) interventions had significant heterogeneity for all-cause mortality; and none of 4 interventions had significant heterogeneity for NEC-related mortality.

\section{AMSTAR Score for Quality of Included Reviews}

The quality of included reviews was adequate. Six included reviews were judged to be of the highest quality (with scores 11 out of 11), 79 were judged to be of "high quality" (with scores ranging from 8 to 10 out of 11), and 13 to be of "medium quality" (with scores ranging from 4 to 7 out of 11). Inappropriate combining of studies and presence or suspicion of publication bias were the 2 most common reasons for downgrading quality of the review. We downgraded any meta-analysis that used a fixed-effects model when high heterogeneity existed $\left(I^{2}\right.$ $>50 \%, p$ for chi square $<0.10$ ). Assessing publication bias was not applicable if a review had $<10$ RCTs. Online supplementary Table 2 provides a breakdown of AMSTAR scores.

\section{GRADE Assessment of Certainty of Evidence}

In all, 10 of 145 interventions provided evidence of moderate certainty for the specified outcomes with the GRADE classification. Other 135 interventions provided evidence of low or very-low certainty and none evidence of high certainty for specified outcomes. In terms of certainty of evidence for each outcome, 7 of 110 interventions, 1 of 10 interventions, and 2 of 21 interventions were rated as moderate certainty for NEC, surgical NEC and all-cause mortality, respectively. No intervention provided moderate certainty for NEC-related mortality. Online supplementary Tables 3-8 represent summary of finding tables of GRADE assessment for each outcome. Although all the interventions were from RCTs, the certainty of evidence was downgraded because of risk of bias, inconsistency, imprecision, or publication bias. 


\section{Discussion}

\section{Principal Findings and Possible Explanations}

Many interventions for preventing NEC have been evaluated, but a comprehensive overview to inform the bedside clinician for evidence-based practice does not exist. This is the first overview of systematic reviews of RCTs of interventions to prevent NEC. We summarized existing evidence from 98 systematic reviews of RCTs, which included antenatal and postnatal interventions that reported NEC as a primary or secondary outcome in preterm infants (online suppl. Table 9). Moderate certainty evidence from our overview showed that the risk of NEC was decreased by probiotics (a combination of species), antenatal corticosteroids for women at risk of preterm birth, probiotics (any), and ibuprofen versus indomethacin for the treatment of PDA. For surgical NEC, lower (85-89\%) oxygen saturation target levels increased the risk, compared with higher (91-95\%) target ranges. A combination of species of probiotics and any probiotics reduced the risk of "all-cause mortality" during hospital stay. No conclusions could be drawn for NEC-related mortality due to lack of data.

Overabundance of pathogenic organisms and lack of microbial diversity in gastrointestinal tract of preterm infants have been reported as key causative factors of NEC. Our findings imply that administration of probiotics decreases NEC and "all cause" mortality possibly by adjusting the microbiome imbalance. The reduced risk of NEC associated with probiotics may also be due to increasing breastfeeding in the probiotics arm of the trials. The certainty of evidence for the use of single species of probiotics is low (Lactobacillus, online suppl. Fig 2) or very low (Bifidobacterium or Saccharomyces boulardii, online suppl. Fig. 3). Unanswered questions for the use of probiotics in preterm infants include selecting the optimal probiotic agent or their combinations, time of initiation, dose, and duration of use.

The certainty of evidence for feeding strategies and type of milk is low to very low including exclusive human milk versus exclusive preterm formula, higher versus lower dose human milk intake, formula milk (term or preterm) versus donor breast milk (unfortified or fortified), fortified breast milk versus unfortified breast milk, hydrolyzed versus nonhydrolyzed formula, protein supplementation of human milk, unpasteurized versus pasteurized human milk, higher versus lower protein intake in formula-fed, slow (up to $24 \mathrm{~mL} / \mathrm{kg} /$ day) versus faster rates of advancement of enteral feeds, delayed ( $\geq 4$ day) versus early introduction of progressive enteral feeding

Preventing NEC in Preterm Infants: An Overview
(24 mL/kg/day), transpyloric versus gastric tube feeding, early trophic feeding versus enteral fasting, and continuous nasogastric milk feeding versus intermittent bolus milk feed (online suppl. Fig. 2, 3).

The outcomes of surgical NEC, "all-cause" mortality, and NEC-related mortality were reported only in a few of the included reviews. Relative effects for substantial interventions were often from only 1 or 2 trials and hence it was difficult to draw firm conclusions for most interventions due to the low or very low certainty of evidence.

\section{Strengths and Limitations of this Overview}

The AMSTAR score is widely accepted as an effective and reliable tool for assessing the quality of systematic reviews. In this overview, the majority of included reviews were of "highest quality" or "high quality." This is partly because majority of included systematic reviews (66 of 98) were published by the Cochrane Collaboration. Notably, high quality of reviews does not equate to high certainty of the evidence of outcome or high quality of the original studies.

We rated the GRADE certainty of evidence ourselves for consistency even if the original review reported it. The certainty of evidence was at best moderate with the majority of evidence being low or very low certainty. Most frequent reasons for downgrading the certainty were high risk of selection bias (lack of blinding and allocation concealment), imprecision (small sample sizes and/or wide CIs), and suspicion or presence of publication bias. Low to very low certainty of evidence call for more methodologically sound, high-quality studies with multicenter collaboration (if possible international), prospective meta-analysis of ongoing trials, and facilitating publication of trials with negative results. This will help in not downgrading the certainty for high risk of selection bias (lack of blinding and allocation concealment), imprecision (small sample sizes and/or wide CIs), and suspicion or presence of publication bias.

An important limitation of this overview is the definition of NEC, which is based on Bell's staging based on clinical and radiologic criteria rather than intestinal pathology or etiology. Since other diseases may have similar clinical or radiological presentation as NEC, an accurate diagnosis is not possible in all patients. For example, clinical and radiological signs of stage I NEC are nonspecific and may be confused with sepsis or feeding intolerance. Surgical NEC is difficult to differentiate from spontaneous intestinal perforation in infants with intraperitoneal air. Even for abdominal X-rays findings in stage II NEC, there is high observer variability to distinguish pneuma- 
tosis in the intestinal wall from fecal bubbles [34]. The assessment of "all-cause mortality," especially where NEC is reported as the secondary outcome should be also be interpreted with caution as other factors including the primary condition may contribute to the mortality.

Our umbrella review is a secondary review of systematic reviews, where we summarize existing evidence rather than creating new evidence and has the limitations of secondary analyses of evidence. We strived to minimize potential biases in this review process. First, an overview protocol was registered with the website PROSPERO (Reg. No. CRD42018105634), https://www.crd.york. ac.uk/PROSPERO/display_record.php?Record ID=105634). Second, 2 authors (T.X. and M.P.) independently searched the databases, collected data, and assessed quality of the included systematic reviews by AMSTAR SCORE (T.X. and A.E.-S.), and assessed certainty of evidence for the outcomes by GRADE (T.X. and M.P.). This provided consistency and reliability of our overview. A potential source of bias is one overview author (M.P.) is the author of one included reviews [15]. A.E.-S., who was not an author of this review, performed the assessment instead of M.P. We only included systematic reviews published in English and we may have missed reviews published in other languages.

Our assessment for the certainty of interventions using the GRADE tool was based on relatively stringent set of criteria. If there were $<10$ trials for a particular intervention, a high likelihood of publication bias was presumed. Our approach was consistent with the recommendations of GRADEprofiler, which advocates that authors of systematic reviews consider downgrading the certainty of evidence by one level when they are not certain about the magnitude of the effect due to selective publication of studies. Our overview targeted systematic reviews that included RCTs as these studies are likely to have the least bias as compared to reviews of observational studies. To get the maximum and up-to-date evidence, we included not only Cochrane reviews but also other reviews containing RCTs. Alternative or emerging strategies for prevention of NEC may not yet have been assessed in reviews of RCTs.

Risk factors of NEC may include antenatal interventions [35]. One strength of our overview is including antenatal interventions that may influence the outcome of NEC. We found that antenatal corticosteroids, prescribed for accelerating fetal lung maturation in women at risk of preterm birth, reduced the risk of NEC (moderate certainty evidence) [28]. Interestingly, nonpharmacological intervention such as oxygen saturation target levels influ- enced the outcome of surgical NEC [33]. Considering the multifactorial etiology of NEC, a multipronged strategy including pharmacological and nonpharmacological interventions is likely to have the best outcome.

\section{Implications for Practice and Research}

Despite a large number of interventions, none had high certainty of evidence for NEC prevention. Decreased risk of NEC was observed with probiotics (combination of species), by antenatal corticosteroids for women at risk of preterm birth, and ibuprofen versus indomethacin for the treatment of PDA. Lower (85-89\%) oxygen saturation target levels may increase the risk of surgical NEC, compared with higher (91-95\%) level. Probiotics, a combination of species or any, decreases the risk of all-cause mortality during hospital stay. We highlight that for most interventions the evidence was of low or very low certainty and statistically significant summary estimates are more likely with such low certainty evidence.

An important consideration is that most studies on NEC are underpowered to detect a clinically significant difference. Studies on NEC (Bell stage $>2$ ), especially on surgical NEC (Bell stage $>3$ ), require large sample sizes to achieve adequate power. The optimal information size that is required for a definite conclusion to be drawn for an intervention to prevent NEC (assuming a baseline NEC incidence of $5 \%$, for $80 \%$ power, alpha of 0.05 ) for a $20 \%$ relative risk reduction, the number of patients required is over 10,000 (Trial sequential analysis software, Copenhagen Trial Unit, Centre for clinical intervention and research, Copenhagen, Denmark, http://www.ctu. $\mathrm{dk} /$ tools-and-links/trial-sequential-analysis.aspx). The large sample sizes call for large multicenter trials, prospective registry and international collaboration for definite conclusions on effectiveness of these interventions.

\section{Statement of Ethics}

The authors have no ethical conflicts to disclose.

\section{Disclosure Statement}

The authors have no financial or conflicts of interest to declare.

\section{Funding Sources}

None. 


\section{Author Contribution}

All authors contributed substantially to this systematic review. T.X. and M.P.: conceptualized the overview of systematic review, performed the search, screened reviews for inclusion, assessed the full text, extracted the data, performed the analysis, and drafted and revised the manuscript. A.E.-S.: assisted in the searches and performed methodological assessment of the included systematic reviews. J.N. and A.M.: provided critical intellectual input during the design of the overview, analysis, drafting, and revising the manuscript. All authors have seen and approved the final version of the report.

\section{References}

1 Neu J, Walker WA. Necrotizing enterocolitis. N Engl J Med. 2011 Jan;364(3):255-64.

2 Sadeghirad B, Morgan RL, Zeraatkar D, Zea AM, Couban R, Johnston BC, et al. Human and Bovine Colostrum for Prevention of Necrotizing Enterocolitis: A Meta-analysis. Pediatrics. 2018 Aug;142(2):e20180767.

3 Warner BB, Deych E, Zhou Y, Hall-Moore C, Weinstock GM, Sodergren E, et al. Gut bacteria dysbiosis and necrotising enterocolitis in very low birthweight infants: a prospective case-control study. Lancet. 2016 May; 387(10031):1928-36.

4 Oddie SJ, Young L, McGuire W. Slow advancement of enteral feed volumes to prevent necrotising enterocolitis in very low birth weight infants. Cochrane Database Syst Rev. 2017 Aug;8(8):CD001241.

5 Chen Y, Chang KT, Lian DW, Lu H, Roy S, Laksmi NK, et al. The role of ischemia in necrotizing enterocolitis. J Pediatr Surg. 2016 Aug;51(8):1255-61.

6 Sisk PM, Lambeth TM, Rojas MA, Lightbourne T, Barahona M, Anthony E, et al. Necrotizing Enterocolitis and Growth in Preterm Infants Fed Predominantly Maternal Milk, Pasteurized Donor Milk, or Preterm Formula: A Retrospective Study. Am J Perinatol. 2017 Jun;34(7):676-83.

7 Pammi M, Cope J, Tarr PI, Warner BB, Morrow $\mathrm{AL}$, Mai $\mathrm{V}$, et al. Intestinal dysbiosis in preterm infants preceding necrotizing enterocolitis: a systematic review and metaanalysis. Microbiome. 2017 Mar;5(1):31.

8 Ganapathy V, Hay JW, Kim JH. Costs of necrotizing enterocolitis and cost-effectiveness of exclusively human milk-based products in feeding extremely premature infants. Breastfeed Med. 2012 Feb;7(1):29-37.

9 Maffei D, Schanler RJ. Human milk is the feeding strategy to prevent necrotizing enterocolitis! Semin Perinatol. 2017 Feb;41(1): 36-40.

10 Sun J, Li Y, Pan X, Nguyen DN, Brunse A, Bojesen AM, et al. Human Milk Fortification with Bovine Colostrum Is Superior to Formula-Based Fortifiers to Prevent Gut Dysfunction, Necrotizing Enterocolitis, and Systemic Infection in Preterm Pigs. JPEN J Parenter Enteral Nutr. 2019 Feb;43(2):252-62.

11 Morgan J, Bombell S, McGuire W. Early trophic feeding versus enteral fasting for very preterm or very low birth weight infants. Cochrane Database Syst Rev. 2013 Mar; 3(3):CD000504.
12 Sawh SC, Deshpande S, Jansen S, Reynaert CJ, Jones PM. Prevention of necrotizing enterocolitis with probiotics: a systematic review and meta-analysis. PeerJ. 2016 Oct; 4:e2429.

13 Johnson-Henry KC, Abrahamsson TR, Wu RY, Sherman PM. Probiotics, Prebiotics, and Synbiotics for the Prevention of Necrotizing Enterocolitis. Adv Nutr. 2016 Sep;7(5):92837.

14 Bury RG, Tudehope D. Enteral antibiotics for preventing necrotizing enterocolitis in low birthweight or preterm infants. Cochrane Database Syst Rev. 2001;1(1):CD000405.

15 Pammi M, Suresh G. Enteral lactoferrin supplementation for prevention of sepsis and necrotizing enterocolitis in preterm infants. Cochrane Database Syst Rev. 2017 Jun; 6(6):CD007137.

16 Pammi M, Haque KN. Pentoxifylline for treatment of sepsis and necrotizing enterocolitis in neonates. Cochrane Database Syst Rev. 2015 Mar;3:CD004205.

17 Foster JP, Seth R, Cole MJ. Oral immunoglobulin for preventing necrotizing enterocolitis in preterm and low birth weight neonates. Cochrane Database Syst Rev. 2016 Apr; 4(4):CD001816.

18 Garg BD, Kabra NS. Role of amino acid supplementation in the prevention of necrotizing enterocolitis in preterm neonates - a review of current evidences. J Matern Fetal Neonatal Med. 2018 Sep;31(17):2349-66.

19 Ardell S, Pfister RH, Soll R. Animal derived surfactant extract versus protein free synthetic surfactant for the prevention and treatment of respiratory distress syndrome. Cochrane Database Syst Rev. 2015 Aug; 8(8):CD000144.

20 Flenady V, Reinebrant HE, Liley HG, Tambimuttu EG, Papatsonis DN. Oxytocin receptor antagonists for inhibiting preterm labour. Cochrane Database Syst Rev. 2014 Jun; 6(6):CD004452.

21 Bond DM, Middleton P, Levett KM, van der Ham DP, Crowther CA, Buchanan SL, et al. Planned early birth versus expectant management for women with preterm prelabour rupture of membranes prior to 37 weeks' gestation for improving pregnancy outcome. Cochrane Database Syst Rev. 2017 Mar; 3:CD004735.

22 Keenan JD, Ayele B, Gebre T, Moncada J, Stoller NE, Zhou Z, et al. Ribosomal RNA evidence of ocular Chlamydia trachomatis in- fection following 3 annual mass azithromycin distributions in communities with highly prevalent trachoma. Clin Infect Dis. 2012 Jan; 54(2):253-6.

23 Bell MJ, Ternberg JL, Feigin RD, Keating JP, Marshall R, Barton L, et al. Neonatal necrotizing enterocolitis. Therapeutic decisions based upon clinical staging. Ann Surg. 1978 Jan; 187(1):1-7.

24 Shea BJ, Hamel C, Wells GA, Bouter LM, Kristjansson E, Grimshaw J, et al. AMSTAR is a reliable and valid measurement tool to assess the methodological quality of systematic reviews. J Clin Epidemiol. 2009 Oct;62(10): 1013-20.

25 Shepherd E, Salam RA, Middleton P, Han S, Makrides M, McIntyre S, et al. Neonatal interventions for preventing cerebral palsy: an overview of Cochrane Systematic Reviews. Cochrane Database Syst Rev. 2018 Jun; 6(6):CD012409.

26 Guyatt GH, Oxman AD, Vist G, Kunz R, Brozek J, Alonso-Coello P, et al. GRADE guidelines: 4 . Rating the quality of evidencestudy limitations (risk of bias). J Clin Epidemiol. 2011 Apr;64(4):407-15.

27 Senn SJ. Overstating the evidence: double counting in meta-analysis and related problems. BMC Med Res Methodol. 2009 Feb; 9(1):10.

28 Roberts D, Brown J, Medley N, Dalziel SR. Antenatal corticosteroids for accelerating fetal lung maturation for women at risk of preterm birth. Cochrane Database Syst Rev. 2017 Mar;3:CD004454.

29 Ohlsson A, Walia R, Shah SS. Ibuprofen for the treatment of patent ductus arteriosus in preterm or low birth weight (or both) infants. Cochrane Database Syst Rev. 2015 Feb; 2(2):CD003481.

30 Doyle LW, Ehrenkranz RA, Halliday HL. Early ( $<8$ days) postnatal corticosteroids for preventing chronic lung disease in preterm infants. Cochrane Database Syst Rev. 2014 May; 5(5):CD001146.

31 Ohlsson A, Aher SM. Early erythropoietin for preventing red blood cell transfusion in preterm and/or low birth weight infants. Cochrane Database Syst Rev. 2014 Apr; 4(4):CD004863.

32 Fowlie PW, Davis PG, McGuire W. Prophylactic intravenous indomethacin for preventing mortality and morbidity in preterm infants. Cochrane Database Syst Rev. 2010 Jul; 7(7):CD000174. 
33 Askie LM, Darlow BA, Finer N, Schmidt B, Stenson B, Tarnow-Mordi W, et al.; Neonatal Oxygenation Prospective Meta-analysis (NeOProM) Collaboration. Association Between Oxygen Saturation Targeting and Death or Disability in Extremely Preterm Infants in the Neonatal Oxygenation Prospective Meta-analysis Collaboration. JAMA. 2018 Jun;319(21):2190-201.

34 Bazacliu C, Neu J. Pathophysiology of Necrotizing Enterocolitis: an Update. Curr Pediatr Rev. 2019;15(2):68-87.

35 Rose AT, Patel RM. A critical analysis of risk factors for necrotizing enterocolitis. Semin Fetal Neonatal Med. 2018 Dec;23(6):374-9.

36 Shah PS, Shah VS, Kelly LE. Arginine supplementation for prevention of necrotising enterocolitis in preterm infants. Cochrane Database Syst Rev. 2017 Apr 11;4(4):CD004339.

37 Silano M, Milani GP, Fattore G, Agostoni C. Donor human milk and risk of surgical necrotizing enterocolitis: A meta-analysis. Clin Nutr. 2019 Jun;38(3):1061-6.

38 Rees CM, Hall NJ, Fleming P, Eaton S. Probiotics for the prevention of surgical necrotising enterocolitis: systematic review and meta- analysis. BMJ Paediatr Open. 2017 Nov; 1(1):e000066.

39 Shah PS, Kaufman DA. Antistaphylococcal immunoglobulins to prevent staphylococcal infection in very low birth weight infants. Cochrane Database Syst Rev. 2009 Apr 15;(2):CD006449.

40 Soghier LM, Brion LP. Cysteine, cystine or Nacetylcysteine supplementation in parenterally fed neonates. Cochrane Database Syst Rev. 2006 Oct 18;(4):CD004869.

41 Miller J, Tonkin E, Damarell RA, McPhee AJ, Suganuma M, Suganuma H, et al. A systematic review and meta-analysis of human milk feeding and morbidity in very low birth weight infants. Nutrients. 2018 May; 10(6):E707.

42 Kenyon S, Boulvain M, Neilson JP. Antibiotics for preterm rupture of membranes. $\mathrm{Co}-$ chrane Database Syst Rev. 2013 Dec 2;(12):CD001058.

43 Zhang P, Lavoie PM, Lacaze-Masmonteil T, Rhainds M, Marc I. Omega-3 long-chain polyunsaturated fatty acids for extremely preterm infants: a systematic review. Pediatrics. 2014 Jul;134(1):120-34.
44 Mosalli R, Alfaleh K, Paes B. Role of prophylactic surgical ligation of patent ductus arteriosus in extremely low birth weight infants: systematic review and implications for clinical practice. Ann Pediatr Cardiol. 2009 Jul; 2(2):120-6.

45 Keir A, Pal S, Trivella M, Lieberman L, Callum J, Shehata N, et al. Adverse effects of red blood cell transfusions in neonates: a systematic review and meta-analysis. Transfusion. 2016 Nov;56(11):2773-80.

46 Livingston MH, Shawyer AC, Rosenbaum PL, Williams C, Jones SA, Walton JM. Glycerin enemas and suppositories in premature infants: a meta-analysis. Pediatrics. 2015 Jun; 135(6):1093-106

47 Osborn DA, Paradisis M, Evans N. The effect of inotropes on morbidity and mortality in preterm infants with low systemic or organ blood flow. Cochrane Database Syst Rev. 2007 Jan 24;1(1):CD005090.

48 Egarter C, Leitich H, Husslein P, Kaider A, Schemper M. Adjunctive antibiotic treatment in preterm labor and neonatal morbidity: a meta-analysis. Obstet Gynecol. 1996 Aug; 88(2):303-9. 